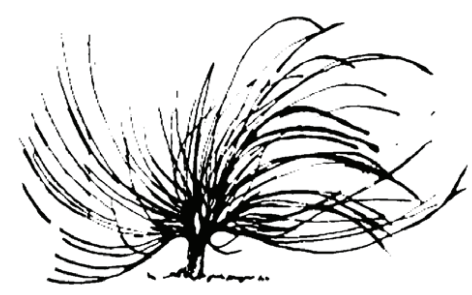

\title{
José Martí, precursor de la pedagogía nuestroamericana
}

\author{
Carla Wainsztok ${ }^{1}$ \\ Universidad de Buenos Aires, Argentina \\ Buenos Aires, Argentina \\ carlalatina@gmail.com
}

\begin{abstract}
Resumen
La idea de este trabajo es reflexionar sobre los aportes de José Martí a la construcción de una pedagogía nuestroamericana. Si bien es cierto que hay muchas experiencias concretas, variadas y muy ricas respecto a las prácticas educativas latinoamericanas, hay un déficit respecto a leerlas en clave de una matriz de pensamiento propia. Con esto queremos decir que la sistematización se presenta en forma fragmentaria y particular; lo cual complejiza interpretarlas dentro un relato propio. Para ello, en este ensayo avanzamos en una definición de una pedagogía latinoamericana.
\end{abstract}

Palabras clave: Pedagogía, Nuestra América, Praxis, José Martí.

\begin{abstract}
The main idea of this work is to reflect on José Martí's contributions to OurAmerican pedagogy. It is known that there are a lot of concrete, varied and rich experiences regarding Latin American teaching practices; however, there is a lack of reading interest in order to create our own thinking matrix. That is, the systematization of practices
\end{abstract}

Recibido: 6 de julio de 2011 - Aprobado: 26 de marzo de 2012

1 Licenciada en Sociología de la Universidad de Buenos Aires, Argentina. Profesora adjunta de Teoría Social Latinoamericana de la Facultad de Ciencias Sociales de esa universidad. Se especializa en Descolonización pedagógica y Pedagogía Social. 
is fragmented and particular which hardens the interpretation of a proper account. In this essay, we move toward a Latin American pedagogy definition.

Keywords: Pedagogy, Our America, Praxis, José Martí.

\section{Pedagogía martiana}

$\mathrm{L}$

a idea de este trabajo es reflexionar sobre la potencialidad de las pedagogías nuestroamericanas. Entendiendo por potencialidad

"un modo de organizar la reflexión sobre el sujeto humano, cuyo principal desafío es romper los parámetros, o lo que se ha definido también como las convenciones que impiden vernos a nosotros mismos en todas nuestras posibilidades" (Zemelman, 2007, p. 9).

Como toda pedagogía, "que reflexiona sobre las formas de transmisión de la cultura, debe exigírsele rigor y coherencia a la hora de construir discursos propios e inteligibles que puedan ser compartidos y rebatidos por estudiosos, profesores, investigadores e interesados en las diferentes dimensiones de la educación. Pero no en menor medida, a la pedagogía le corresponde -independientemente del adjetivo que la acompaño- trabajar para generar y consolidar nuevas modalidades de explicar, enseñar, transmitir conocimientos y saberes que la época y el lugar donde se desarrolla consideran valiosos" (García Molina, 2003, p. 52).

Estos son tiempos latinoamericanos y no por capricho de algún investigador/a son tiempos indoamericanos por voluntad de los pueblos. Por ello, la definición de una pedagogía nuestroamericana debe incluir tres temas la formación, la generación y la transmisión.

Es necesaria una formación de y en nuestros contenidos, sin perjuicio de conocer el universal, "La historia de América, de los incas hasta acá, ha de enseñarse al dedillo, aunque no se enseñe la de los arcontes de Grecia. Nuestra Grecia es preferible a la Grecia que no es nuestra. Nos es más necesaria (...) Injértese en nuestras Repúblicas el mundo, pero el tronco ha de ser de nuestras Repúblicas" (Martí, 2005, p.12).

Pero aún más una pedagogía latinoamericana no lo es por un tema geográfico sino por su interpretación en clave geopolítica. Se trata de leer desde el Sur, construir una epistemología del Sur, entendiendo por esta "la búsqueda de conocimiento y de criterios de validez del conocimiento que otorguen visibilidad y credibilidad a las prácticas cognitivas 
de las clases, los pueblos y de los grupos sociales que han sido históricamente victimizados, explotados y oprimidos, por el colonialismo y el capitalismo globales. El Sur, es, pues, usado aquí como metáfora del sufrimiento humano sistemáticamente causado por el colonialismo y el capitalismo" (De Sousa Santos, 2009, p.12).

En relación con las generaciones, la enseñanza y la reflexión sobre esta es una promesa con las nuevas generaciones. Pero incluso en nuestros países con historias de exilios, destierros, reunir las viejas y las nuevas generaciones en un relato no es un dato menor. De ello también se nutre la pedagogía, como diálogo intergeneracional. Las dictaduras destruyeron la posibilidad de ese diálogo; que hoy se está reconstruyendo. Entonces la pedagogía nuestroamericana se constituye también como filiación simbólica. Es ahí donde generación y transmisión se dan la mano. Transmisión no como algo mecánico, una "concepción bancaria" sino como la posibilidad de compartir relatos. En Nuestra América la opresión es doble, expoliación económica y colonización cultural. Nos han privado de nuestras cuentas y nuestros cuentos.

Respecto a Martí "la idea de hombre que defendió adelantaba una visión dialéctica en búsqueda constante del desarrollo de la propia naturaleza humana. Para lograr este propósito concibió un sistema de formación integral donde debían combinarse los procesos de conocer, pensar, apreciar y actuar como vías para formar principios y valores. El núcleo central de tal espiral era la formación de las emociones y los sentimientos" (Pérez Cruz, : 2002, p.13).

Su concepción filosófica es relacional, es decir, no hay falsas dicotomías sino que en su pedagogía conviven la idea de revolución, materialismo, espiritualismo y patriotismo. "Patriotismo es amor entrañable a la nación y conciencia del deber para con su desarrollo y defensa. Ello está profundamente interconectado con el culto y el respeto martiano a la libertad, la justicia, el decoro de los hombres, valores que resume en el concepto de dignidad de los pueblos" (Pérez Cruz, 2002,p.14).

Y en sintonía con la formación y lo generacional:

El aporte más trascendental de José Martí estuvo precisamente en concebir la escuela y el maestro en el contexto más amplio de la sociedad para contribuir de modo eficaz al objetivo de preparar al hombre para la vida y ponerlo en consonancia con su pueblo y su 
tiempo. Tal cosmovisión superó definitivamente el paradigma liberal al concebirse la labor educativa como proceso de formación revolucionaria de las nuevas generaciones, de los trabajadores y el pueblo, como acto de transformación emancipadora de las personas y sus circunstancias". (Pérez Cruz; 2002, p. 21).

Sin embargo, no debemos olvidar que Martí sospecha de la pedagogía "y en las clases mismas, como en lo hablado y escrito por los hijos de la casa, se ve la fuerza y realidad de aquella gente generosa. Están a lo útil y no a lo ornamental: a los resultados, y no a las pedagogías" (Martí, 2001, p. 268).

Podemos aventurar que el recelo de Martí es hacia los pedagogos profesionales, los que hacen de la tarea de enseñar un mero formalismo. "Hay que crear, si escuelas normales; pero no escuelas normales de pedantes, de retóricos, de nominalistas, sino maestros vivos" (Martí, 1985, p.14).

Un maestro escribe "en las almas, que suelen olvidar" (Martí, 2001, p. 248) pero además la letra pedantesca también puede ser hallada en los libros, entonces contra los pedagogos y los escritores pedantes.

Nosotras/os por nuestra parte sabemos que existen otras corrientes pedagógicas y queremos aportar a una construcción propia a las pedagogías de Nuestra América por ello "no quisiéramos construir una pedagogía que, como siempre, refleje los avances ocurridos en otros países, bajo otra historia, otras condiciones sociales, otras prospectivas. Si pensamos que hay que analizar esos aportes, interpretándolos en función de nuestra particular historia" (Silber, 2000, p.118).

Los conceptos que humildemente y junto a otras compañeras y compañeros podemos aportar no descienden del cielo a la tierra, ni salen de cavernas oscuras, ellas provienen de las prácticas sociales. De nuestras prácticas sociales. En este caso de nuestras prácticas pedagógicas concretas.

Para poder colaborar en la construcción de nuestras pedagogías es necesario sistematizar las prácticas de las maestras y los maestros que nos han precedido. Si toda pedagogía, como ya hemos mencionado supone un enlace entre generaciones, el rescate de nuestras pedagogías no es un asunto museológico, necesitamos de las "viejas voces" para que junto a las "nuevas prácticas" se fusionen en legados y creaciones. 
La transmisibilidad no es, en efecto, algo externo y mecánico, sino que es una posibilidad intrínseca de toda herencia cultural que explica que puede ser recibida. Las formas culturales son necesariamente informadas y conformadas en mayor o menor grado por quien las recibe, caso contrario no se puede hablar de recepción y ello deriva de su propia naturaleza histórica (Roig; 2009:49).

Además, más allá de toda clase de artificios, la pedagogía está constituida por teorías. Heredamos y construimos conceptos.

\section{Los maestros de Martí}

Para el filósofo George Steiner es posible "distinguir tres escenarios principales o estructuras de relación. Hay Maestros que han destruido a sus discípulos psicológicamente y, en algunos raros casos, físicamente. Han quebrantado su espíritu, han consumido sus esperanzas, se han aprovechado de su dependencia y de su individualidad. El ámbito del alma tiene sus vampiros. Como contrapunto, ha habido discípulos, pupilos y aprendices que han tergiversado, traicionado y destruido a sus Maestros (...) La tercera categoría es la del intercambio, el eros de la mutua confianza e incluso el amor" (Steiner, 2005, p.12).

En esta última estructura es donde podemos ubicar a Martí y sus maestros. El Apóstol de Nuestra América, reconoce a dos maestros, a José de la Luz a quien no conoció personalmente y, a Rafael Mendive. "Pero si José de la Luz fue leyenda, Mendive constituyó el ejemplo cotidiano de un poeta y un maestro" (Nassif, 1999, p.1).

José de la Luz "había sido el maestro de la generación anterior a Martí" (Nassif, 1999, p1) y sobre este dirá el aprendiz: "Supo cuanto se sabía en su época, pero no para enseñar que lo sabía, sino para transmitirlo. Sembró hombres" (Martí, 2001, p. 248).

"Martí fue fruto de la educación patriótica que ya a mediados del siglo XIX había echado raíces en la mayor isla del Caribe. Estudió las obras y bebió en la tradición oral aún fresca de los precursores de la escuela cubana, de Félix Várela y José de la Luz y Caballero" (Pérez Cruz, 2002, p. 9).

En relación con Mendive, Martí afirmaba: ¿y cómo quiere que en algunas líneas diga todo lo bueno y nuevo que pudiera yo decir de aquel 
enamorado de la belleza, que la quería en las letras como en las cosas de la vida, y no escribió jamás sino sobre verdades de su corazón o sobre penas de la patria? “(Martí, 2001, p. 250) .

Creemos que es pertinente en la producción de las pedagogías latinoamericanas escribir las relaciones entre maestros y discípulos. En este sentido el eros pedagógico entre Martí y Mendive nos recuerda la relación de Bolívar y Rodríguez.

Bolívar y Martí recuerdan con emoción las lecciones de sus maestros y esto nos convoca a pensar nosotros en estas relaciones con nuestras/os maestras/os. ¿Qué nos enseñaron? ¿Qué nos han legado? ¿Qué hemos recibido? En principio, la alegría del aprender y enseñar. En este sentido no hay docencia sin alegría ni sin esperanzas.

\section{Un maestro de Nuestra América Latina}

El apóstol de América nace en 1853 en Cuba y muere defendiendo la independencia de su territorio en la batalla de Dos Ríos en 1895. "Su inicio profesional en la enseñanza tuvo lugar enGuatemala, en 1877, cuando recién llegado fue contratado por elgobierno de ese país para impartir clases en la Escuela Normal, (...) En la Escuela Normal, Martí fue profesor de literatura y composición. Al mes siguiente fue nombrado también catedrático de Literatura yde Historia de la Filosofía en la Facultad de Filosofía y Letras enla Universidad Central. Y su empeño docente también lo llevará a ofrecer clases gratuitas de composición en la Academia de Niñas de Centro América." ( Pérez Cruz; 2002, p.11).

Luego de una conspiración a favor de la Independencia de Cuba, fue encarcelado por segunda vez, para posteriormente partir al exilio. "Venezuela lo recibe en 1881 y allí, a poco de llegar, el Colegio de Santa María le encarga las clases de lengua y literatura francesa" (Nassif, 1999, p.2). En 1899, participa en la Liga de la Instrucción de Nueva

York y como profesor de español en la Central High School.Respecto a La liga de la Instrucción escribió:

'La liga' de Nueva York es una casa de educación y cariños, aunque quién dice educar, ya dice querer. En 'La Liga' se reúnen, después de la fatiga de trabajo, los que saben que sólo hay dicha verdadera en la amistad y en la cultura: los que en sí sienten o 
ven por sí que el ser de un color o de otro no merma en el hombre la aspiración sublime; los que no creen que ganar el pan en un oficio, da al hombre menos derechos y obligaciones que los de quienes lo ganan en cualquier otro (...) los hijos de las dos islas que, en el sigilo de la creación, maduran el carácter nuevo por cuya justicia y práctica firme se ha de asegurar la patria (...) sacarles el espíritu con los fuegos o choques de la conversación, o enseñar a los que saben menos, aprender de los que más se sabe (Martí, 2001, p. 252).

La liga "es el hogar de las ideas que desde hace años pagan, del sacrificio de sus difíciles salarios, unos cuantos obreros cubanos, obreros de color: de esos obreros nuestros". (Martí, 2001, p. 267).Fueron esos obreros los que "lo bautizaron para la historia como Maestro, referían con ello al magisterio mayor que ya había asumido". ( Pérez Cruz, 2002, p. 11).

\section{Ideas pedagógicas}

En un texto que se llama Educación Popular, Martí distingue entre educación e instrucción. La educación se refiere al sentimiento y la instrucción al pensamiento. Esta es en sí misma una definición clásica, la instrucción es individual y tiene que ver con los conceptos y la educación es colectiva y tiene que ver con los valores, pero Martí insiste para que haya buena educación tiene que haber buena instrucción, puesto que "las cualidades morales suben de precio cuando están realizadas por cualidades inteligentes (...) El pueblo más feliz es el que tenga mejor educados a sus hijos, en la instrucción del pensamiento y en la dirección de los sentimientos. Un pueblo instruido ama el trabajo y sabe sacar provecho de él" (Martí, 2001, pp. 375-376).

Además de la distinción antes mencionada nos interesa la relación entre educación y trabajo. En su texto Una escuela de artes y oficios en Honduras sostuvo: "La Escuela de Artes y Oficios es invención muy buena; pero sólo puede tenerse una, y para hacer todo un pueblo nuevo no basta. La enseñanza de la agricultura es aún más urgente; pero no en escuelas técnicas, sino en estaciones de cultivo; donde no se describan las partes del arado sino delante de él y manejándolo; y no se explique en fórmula sobre la pizarra la composición de los terrenos, 
sino en las capas mismas de tierra; y no se entibie la atención de los alumnos con meras reglas técnicas de cultivos, rígidas como las letras de plomo con que se han impreso, sino que se les entretenga con las curiosidades, deseos, sorpresas y experiencias, que son sabroso pago y animado premio de los que se dedican por sí mismos a la agricultura" (Martí, 1980, p. 172).

Martí tiene una consideración positiva del trabajo. El trabajo es entendido como proceso creativo. "El hombre crece con el trabajo que sale de sus manos (...) el que debe su bienestar a su trabajo, o ha ocupado su vida en crear y transformar fuerzas, y en emplear las propias, tiene el ojo alegre, la mano pintoresca y profunda, las espaldas anchas, y la mano segura. Se ve que son ésos los que hacen al mundo; y engrandecidos, sin saberlo acaso, por el ejercicio de su poder de creación, tienen cierto aire de gigantes dichosos, e inspiran ternura y respeto" (Martí, 2001, p. 285).

Y en el texto homenaje a Karl Marx prosigue: "El trabajo embellece. Remoza ver a un labriego, a un herrador, o a un marinero. De manejar las fuerzas de la naturaleza, les viene ser hermosos como ellas" (Martí, 2005, p. 114).Sin embargo, educar para el trabajo no se opone a la educación para la vida.

En 1883, Martí escribe: "Al mundo nuevo corresponde una universidad nueva. (..) Es criminal el divorcio entre la educación que se recibe en una época y la época. Educar es depositar en cada hombre toda la obra humana que le ha antecedido: es hacer de cada hombre resumen del mundo viviente, hasta el día en que vive es ponerlo a nivel de su tiempo, para que flote sobre él y no dejarlo debajo de su tiempo, con lo que no podría salir a flote, es preparar al hombre para la vida" (Martí, 2001, p.281).

Martí a lo largo de su vida no cree en las falsas dicotomías por ello respecto a la educación pudo afirmar:

Y no está la reforma completa en añadir cursos aislados de enseñanza científica a las universidades literarias, sino en crear universidades científicas sin derribar por eso jamás las literarias, en llevar el amor a lo útil y la abominación de lo inútil, a las escuelas de letras; en enseñar todos los aspectos del pensamiento humano en cada problema, y no -con lo que se cometa alevosa traiciónun solo aspecto en llevar solidez científica, solemnidad artística, 
majestad y precisión arquitecturales a la Literatura (...) La literatura de nuestros tiempos es ineficaz, porque no es la expresión de nuestros tiempos (Martí, 2001, p. 283).

Martí reflexiona sobre los nuevos tiempos, "los tiempos están revueltos; los hombres están despiertos" (Martí, 2001, p. 284); “el mundo está de cambio" (Martí, 2001, p. 290).Como puede percibirse hay una gran preocupación respecto a la distancia entre la formación y la época. La hacemos nuestra preocupación.

\section{Martí y los maestros}

En varios de sus textos, Martí reflexiona sobre los maestros, tanto en lo que se refiere a su formación, como a su trabajo. Como afirmamos al comienzo del trabajo: "Hay que crear, si escuelas normales; pero no escuelas normales de pedantes, de retóricos, de nominalistas, sino maestros vivos y útiles que puedan enseñar la composición, riquezas y funciones de la tierra, las maneras de hacerlas producir y de vivir dignamente sobre ella y las noblezas pasadas y presentes que mantienen a los pueblos, preservando en el alma la capacidad y el apetito de lo heroico" (Martí,1985, p. 14).

El maestro cubano duda del saber y los propósitos de enseñar de los pedantes: "No enviaríamos pedagogos por los campos, sino conversadores. Dómines no enviaríamos, sino gente instruida que fuera respondiendo a las dudas que los ignorantes les presentasen o las preguntas que tuviesen preparadas para cuando vinieran (...) En suma, se necesita abrir una campaña de ternura y de ciencia, y crear para ella un cuerpo, que no existe, de maestros misioneros" (Martí, 2001, p. 289).

Maestros misioneros, una primer lectura nos haría desconfiar de esta imagen. La cercanía a un discurso religioso pero también positivista de la docencia como apostolado, lo haría volver refractario, sin embargo es necesario dar batalla semiótica sobre el concepto de misión.

Una definición que nos interesa dentro de la concepción martiana sería obra o función moral que se tiene que realizar por el bien de alguien y en ese punto queremos recordar la siguiente idea: "Al venir a la tierra todo hombre tiene derecho a que se le eduque, y después en pago, el deber de contribuir la educación de los demás" (Martí, 1991, p. 375). 
La idea de Martí consistía en "llevar los maestros por los campos. No sólo explicaciones agrícolas e instrumentos mecánicos, sino la ternura que hace tanta falta y tanto bien a los hombres. El campesino no puede dejar su trabajo para ir sendas millas a ver figuras geométricas incomprensibles, y aprender los cabos y los ríos de las penínsulas del África y proveerse de vacíos términos didácticos. Los hijos de los campesinos no pueden apartarse leguas enteras día tras día de la estancia paterna para ir a aprender declinaciones latinas y divisiones abreviadas. Y los campesinos, sin embargo, son la mejor masa nacional, y la más sana y jugosa, porque recibe de cerca y de llano los efluvios y la amable correspondencia de la tierra en cuyo trato viven. Las ciudades son la mente de las naciones, pero su corazón, donde se agolpa, y de donde se reparte la sangre, está en los campos" (Martí, 2001, p. 290).

La revolución cubana retoma estas ideas de Martí y, en 1960 ante un llamado de Fidel Castro se constituye el movimiento de Maestros Voluntarios. El 22 de diciembre de 1961, se declara a Cuba territorio libre de analfabetismo y, es proclamado el día nacional del educador.

La idea de misión sigue presente en Nuestra América, en la actualidad en Venezuela "las Misiones operan como verdaderas estructuras paralelas a los ministerios, cuya flexibilidad permite satisfacer en un plazo mucho menor las demandas de salud y educación, generando una duplicidad institucional estatal" (Imen, 2010, p. 196).Los nombres de las misiones hacen claras referencias a la historia venezolana: Misión Robinson, Misión Ribas, Misión Negra Hipólita, Misión Sucre.

\section{La Patria es la Infancia}

Entre julio de 1889 y octubre del mismo año Martí es editor de la revista "La Edad de Oro", una revista infantil que aquí tomamos como otra práctica pedagógica martiana.En su título ya se afirma que es una publicación mensual de recreo e instrucción dedicada a los niños de América.

En la editorial del primer número Martí afirma: "Para los niños es este periódico, y para las niñas, por supuesto. Sin las niñas no se puede vivir, como no puede vivir la tierra sin la luz (...) Para eso se publica la EDAD DE ORO, para que los niños americanos sepan cómo se vivía antes y se vive hoy, en América, y en las demás tierras; y como se hacen tantas cosas de cristal y de hierro, y las máquinas de vapor, y los puentes colgantes, y la luz eléctrica (...) Para los niños trabajamos, porque los 
niños son los que saben querer, porque los niños son la esperanza del mundo. Y queremos que nos quieran y nos vean como cosa de su corazón. (...) Así queremos que los niños de América sean: hombres que digan lo que piensan, y lo digan bien: hombres elocuentes y sinceros" (Martí, 1889, p.1).

En ese primer número, la primer nota se llama Tres héroes, en ella Martí les cuenta a los niños y las niñas sobre Bolívar, Hidalgo y San Martín, le sigue la traducción de un cuento (Meñique) y la historia de la Ilíada dando por sentado de que nuestra Grecia es más importante que la Grecia de los Arcontes.

Posteriormente, hay un artículo sobre los juegos, otro cuento y lo que se llama La última página, en la cual puede leerse "Treinta y dos páginas es de veras poco para conversar con los niños queridos, con los que han de ser mañana hábiles como Meñique y valientes como Bolívar, poetas como Homero ya no podrán ser, porque estos tiempos no son como los de antes, y las aedas de ahora no han de cantar guerras bárbaras de pueblo con pueblo para ver cual puede más, ni peleas de hombre con hombre para ver quién es más fuerte. (...) Los versos no se han de hacer para decir que se está contento o se está triste, sino para ser útil al mundo, enseñándole que la naturaleza es hermosa, que la vida es un deber, que la muerte no es fea, que nadie debe estar triste ni acobardarse mientras haya libros en la librería, y luz en el cielo y amigos y madres (...) Antes todo se hacía con los puños, ahora la fuerza está en el saber, más que en los puñetazos, aunque es bueno aprender a defenderse, porque siempre hay gente bestial en el mundo, y porque la fuerza da salud, y porque se ha de estar pronto a pelear, para cuando un pueblo ladrón quiera venir a robarnos nuestro pueblo" (Martí, 1889, p. 32).

Del número dos queremos mencionar, la nota sobre músicos y pintores pues en la misma hay una definición de educación: "Cada ser humano lleva en si un hombre ideal (...) la educación empieza con la vida, y no acaba sino con la muerte. El cuerpo es siempre el mismo, y decae con la edad; la mente cambia sin cesar, y se enriquece y perfecciona con los años. Pero las cualidades esenciales del carácter, lo original y enérgico de cada hombre, se deja ver desde la infancia en un acto, en una idea, en una mirada(...) Lo general es que el hombre no logre en la vida un bienestar permanente sino después de muchos años de esperar con paciencia y de ser bueno, sin cansarse nunca" (Martí, 1889 , p. 57). 
En el último número Martí va anunciando a donde lo llevan sus ideales. "Se ha de conocer las fuerzas del mundo para ponerlas a trabajar (...) pero el hombre ha de aprender a defenderse y a inventar, viviendo al aire libre, y viendo la muerte de cerca (...) Hay que ir de vez en cuando a vivir en lo natural y a conocer la selva" (Martí, 1889, p. 128).

En 1895, Martí le escribe una carta de despedida a su hija María, esta epístola preanuncia otra escrita por otro revolucionario a sus hijos. "Cuando alguien me es bueno y bueno a Cuba, le enseño tu retrato. (...) Espérame, mientras seas que yo viva (...) Enseñar es crecer (...) Yo no recuerdo, entre los que tú puedas tener a mano, ningún libro escrito en este español simple y puro. Yo quise escribir así en La Edad de Oro; para que los niños me entendiesen, y el lenguaje tuviera sentido y música.(...) Estudia, mi María, trabaja y espérame(...) Tengo la vida a un lado de la mesa, y la muerte a otro, y un pueblo a las espaldas; y ve cuántas páginas te escribo (...) Y si no me vuelves a ver, haz como el chiquitín cuando el entierro de Frank Soriano: pon un libro, -el libro que te pido -sobre la sepultura. O sobre tu pecho, porque ahí estaré enterrado yo si muero donde lo sepan los hombres. Trabaja. Un beso. Y espérame" (Martí, 2005, p.156).

Y pensando en esos otros revolucionarios y en términos de generación "No es hasta 1932 cuando por iniciativa de Emilio Roig de Leuchsenring y con prólogo suyo, se vuelve a publicar la Edad de Oro como libro aparte, en lo que resulta su primera edición cubana pues las anteriores habían sido hechas en los Estados Unidos, en Italia (la de Gonzalo de Quesada) u en Costa Rica, auspiciada por el intelectual J. García Monge (...) los niños que leyeron esas reediciones fueron los que al cumplirse el centenario de José Martí, en 1953, estaban ya en la madurez de sus veinte años: es la llamada Generación del Centenario, que contempló como otro tirano sometía a aquella seudorrepública, mientras cínicamente rendía homenajes a un hombre cuyos ideales pisoteaba en la práctica" (Arias, 2007, p. 503).

\section{Nuestra América}

En 1891, hace 120 años, se publicaba el manifiesto Nuestra América. De todos los análisis plausibles vamos a centrarnos en los siguientes ejes: el lugar de las ideas, el conocimiento, la universidad, la razón; 
la falsa dicotomía civilización-barbarie, el odio, las razas; el sujeto: los oprimidos, el hombre nuevo.

En relación con las ideas, Martí sostiene: "Trincheras de ideas valen más que trincheras de piedras", y continúa: "una idea enérgica, flameada a tiempo ante el mundo para, como la bandera mística del juicio final, a un escuadrón de acorazados. Los pueblos que no se conocen han de darse prisa para conocerse como quienes van a pelear juntos" (Martí, 2005, p. 8).

Las ideas, son herramientas, son armas, las ideas son para conocerse y reconocerse. ¿Quiénes han de conocerse? los pueblos. "Ya no podemos ser el pueblo de hojas, que vive en el aire, con la copa cargada de flor (...) es la hora del recuento, y de la marcha unida, y hemos de andar en cuadro apretado, como la plata en las raíces de los Andes" (Martí, 2005, p. 8).

El desconocimiento de nosotros mismos es mentalidad aldeana: "El continente descoyuntado durante tres siglos por un mando que negaba el derecho del hombre al ejercicio de la razón, entró desatiendo o desoyendo a los ignorantes que lo habían ayudado a redimirse, en un gobierno que tenía por base la razón, la razón de todos en las cosas de todos, y no la razón universitaria de uno sobre la razón campestre de otros. El problema de la independencia no era el cambio de formas, sino el cambio de espíritu" (Martí, 2005, p.11).

El problema de la Independencia es entender que "la revolución triunfó con el alma de la tierra (...) con el alma de la tierra había que gobernar y no contra ella ni sin ella". Somos el libro importado y el alma de la tierra, somos el libro importado y las botas de potro, somos alpargatas y libros. "Si la República no abre los brazos a todos y adelanta con todos, muere la República" (Martí, 2005, pp. 11-12).

Estamos frente a una definición ampliada de razón y república. La razón y la república somos todos, "nuestra América mestiza". En Nuestra América mestiza, "conocer es resolver", "pensar es servir" (y el leer es para aplicar "no para copiar" (Martí, J.: 2005, pp. 10, 13-14).

Afirma Martí que "las levitas son todavía de Francia, pero el pensamiento empieza a ser de América. Los jóvenes de América se ponen la camisa al codo, hunden las manos en la masa y la levantan con la levadura de su sudor. Entienden que se imita demasiado, y que la salvación está en crear. Crear es la palabra de pase de esta generación" (Martí, 2005, p. 12). 
Para conocer, pensar, crear es necesario que las instituciones educativas estén como diría Martí acorde a los tiempos “¿Cómo han de salir de las Universidades los gobernantes, si no hay Universidad en América donde se enseñe lo rudimentario del arte del gobierno, que es el análisis de los elementos peculiares de los pueblos de América? A adivinar salen los jóvenes al mundo, con antiparras yanquis o francesas, y aspiran a dirigir un pueblo que no conocen (...) El premio no ha de ser para la mejor oda, sino para el mejor estudio de los factores del país en que se vive (...) La universidad europea debe ceder a la universidad americana". (Martí, 2005, p.12).Creemos que la obra de una universidad latinoamericana es una tarea pendiente.

Para Salvador Allende, las escuelas fronterizas y la universidad latinoamericana permitirían construir el Estatuto del hombre latinoamericano. Un estatuto descolonizador donde no podrían ingresar las falsas dicotomías.

"No hay batalla entre civilización y barbarie, sino entre falsa erudición y la naturaleza. El hombre natural es bueno, y acata y premia la inteligencia superior, mientras ésta no se vale de su sumisión para dañarle, o le ofende prescindiendo de él, que es cosa que no perdona el hombre natural, dispuesto a recobrar por la fuerza el respeto de quien le hiere la susceptibilidad o le perjudica el interés" (Martí, 2005, p. 10).Sobre el desdén del hombre natural "han subido los tiranos de América al poder; y han caído en cuanto les hicieron traición” (Martí, 2005, p.10).

Contra este menosprecio del hombre natural es necesario "un conocimiento situado, que demanda una atención continua a la identidad, la conducta y al involucramiento en la vida pública (...) Nuestra América conlleva así un fuerte componente epistemológico. En vez de importar ideas extranjeras, uno debe buscar las realidades específicas del continente desde una perspectiva latinoamericana" (De Sousa Santos, 2009, p. 229).

Es en este sentido que "ni el libro europeo, ni el libro yanqui, daban la clave del enigma hispanoamericano. Se probó el odio, y los países venían cada año a menos. Cansados del odio inútil, de la resistencia del libro contra la lanza, la razón contra el cirial, de la ciudad contra el campo (...) se empieza, como sin saberlo a probar el amor" (Martí, 2005, p.12).

Es interesante la relación entre libro europeo, yanqui y el odio, Martí nos convida a pensar desde nuestro continente y con alegría. Pero 
no es una alegría simplista, no hay alegría más grande que colaborar en la construcción de nuestros relatos.

En Nuestra América, "peca contra la humanidad el que fomente y propague la oposición y el odio de las razas" (Martí, 2005, p. 14).Frente el odio y al exterminio, enfrentados a los "los pensadores canijos, los pensadores de lámparas, enhebran y recalientan las razas de librería” de la vereda de la vida, del respeto, la diversidad y "en pie, con los ojos alegres de los trabajadores se saludan, de un pueblo a otro, los hombres nuevos americanos" (Martí, 2005, pp. 12-13).

Es "significativo que Martí, tan atento siempre a los valores latinoamericanos, no publicara un solo trabajo sobre Sarmiento, ni siquiera a raíz de su muerte en 1888" (Fernández Retamar, 1973, p. 83).

Sarmiento por su parte si habló de Martí: "Una cosa le falta a don José Martí para ser un publicista (...) fáltale regenerarse, educarse, si es posible decirlo, recibiendo del pueblo en que vive la inspiración, como se recibe el alimento para convertirlo en sangre que vivifica (...) Quisiera que Martí nos diera menos Martí, menos español de raza y menos americano del Sur, por un poco más del yankee, el nuevo tipo del hombre moderno" (Fernández Retamar, 1973, p. 90).

Pero son otros los hombre nuevos americanos: son trabajadores, campesinos, indios que conforman nuestra América mestiza son "los oprimidos" (Martí, 2005, p.11) con los cuales "había que hacer causa común, para afianzar el sistema opuesto a los intereses y hábitos de mando de los opresores" (Martí:2005, 1p. 1).

Decíamos que Nuestra América es un texto pedagógico y forma parte de la pedagogía martiana, es una pieza más de este palimpsesto formativo y esto más allá de pensar el conocimiento y la razón.

Nuestra América es un escrito que deviene en programa de acción. Nos indica cuáles son las tareas a realizar en nuestro continente mestizo, con quiénes nos habremos de hermanar y, como buen relato pedagógico nos vincula con la alegría y con el devenir.

Martí nos convida aún hoy a "sembrar escuelas (...) ponerse al nivel del propio tiempo" (Martí, 1980, p.185) y nosotras/os humildemente deseamos sumarnos a la siembra de ideas pedagógicas nuestroamericanas que eventualmente puedan ser cosechadas en el presente y en el futuro por las/los docentes de Nuestra América mestiza. 


\section{Referencias bibliográficas}

Arias, S. (2007) Singularidad y deleite de la revista martiana en José Martí. La Habana: Casa de las Américas.

De Sousa Santos, B. (2009).Una epistemología del SUR. Buenos Aires: CLACSO.

Fernández Retamar, R. (1973) Caliban: apuntes sobre la cultura de Nuestra América. Buenos Aires: La pléyade.

Imen, P. (2010) La escuela pública tiene quien le escriba. Buenos Aires: Centro Cultural de la Cooperación.

Martí, J. (2005) Nuestra América y otros escritos. Buenos Aires: El Andariego.

Martí, J. (2005) Karl Marx en Nuestra América y otros escritos. Buenos Aires: El Andariego.

Martí. J. (1951) Apuntes inéditos. La Habana: Publicaciones Archivo Nacional de Cuba.

Martí. J. (1980) Autores Americanos Aborígenes en Nuestra América. Buenos Aires: Losada

Martí, J. (2001) Obras completas. La Habana: Centro de Estudios Martianos.

Martí, J. (1991) Obras completas. La Habana: Editorial Ciencias Sociales

Martí, J. (1985) Revolución en la enseñanza. La Habana: Anuario del Centro de Estudios Martianos.

Martí, J. (1980) Una escuela de artes y oficios en Honduras en Nuestra América. Buenos Aires: Losada

Martí, J. (1889) La Edad de Oro. Nueva York: Da Costa Gómez.

Nassif, R. (1999) José Martí. Paris:UNESCO.

Pérez Cruz, F. (2002) "El proyecto educativo martiano principios para la praxis en el siglo XXI".Revista Docencia. (6).

Roig, A. (2009) Teoría y crítica del pensamiento latinoamericano. Buenos Aires: Una ventana.

Silber, J. (2000) Acerca de la construcción del campo pedagógico desde el paradigma crítico en Análisis político y propuestas pedagógicas. Buenos Aires: Aique.

Silber, J. (2009) Pedagogía... una disciplina indisciplinada. Universidad Nacional de Rosario.

Steiner, G. (2005) Lecciones de los maestros. México. Siruela.

Zemelman, H. (2007) El ángel de la historia. Barcelona. Anthropos 